\title{
Retention of Fetal Membranes in Aborted Kathiawari Mare: A Case Report
}

\author{
MP Patel*, BS Rathod, HC Nakhashi and BN Suthar \\ S.D. Agricultural University, Department of Gynecology and Obstetrics, College of Veterinary Science and Animal Husbandry, India
}

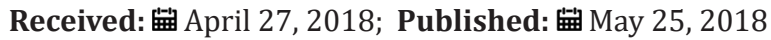

*Corresponding author: MP Patel, S.D. Agricultural University, Department of Gynecology and Obstetrics, College of Veterinary Science and Animal Husbandry, India

Keywords: Cultural Heritage; Global Recognition; Foreign Direct Investment; Dairy Farm; National Dairy Plan; Consumer Class; Milk Processing; Packaging; Marketing Wholesome Milk; Milk Products

\section{Introduction}

Failure to passage a part or all of the allantochorionic membrane with or without the amniotic membranes within a prescribed period of the time after parturition is known as Retention of the Fetal Membranes (RFM). The length of time for expulsion of placenta in mare varies among authors from 30 minutes to 6-12 hours Vandeplassche [1], Blanchard [2] and Provercher [3]. This condition is reported to occur with a frequency of 2 to $10.5 \%$ in draught horses Allen [4]. The portion of the membranes most likely to be retained and the condition occurs more frequently in mares which have undergone abortion, dystocia, embryotomy and caesarean section Vandeplassche [1].

\section{Case History and Clinical Observations}

A fifteen years old Kathiawari mare aborted at 9 months pregnancy was presented to TVCC, Deesa with a time elapse of 8 hours for membrane expulsion. The animal showed the signs of colic along with rapid and shallow respiration, congested mucus membrane and $100 \mathrm{oF}$ rectal temperatures. The fetal membranes were protruding from the vulvar lips to a length of approximately $10 \mathrm{~cm}$. The animal had unusual gait and bear more weight on the rear limbs than the forelimbs. The per-vaginal examination revealed the presence of adhered fetal membranes to the endometrium and the case was diagnosed as post abortion retention of fetal membranes.

\section{Treatment and Discussion}

The mare was secured by hobbling the limbs. The perineum was washed, cleaned and dried. The assistant hold the tail to one side and ointment Barrier (Terpineol B.P. vet 3\% w/w) cream was applied on the gloved hands. The portion of placenta hanging outside the vulva was held with one hand and the placenta was twisted gently in a rope fashion (Figure 1). The other hand was carefully introduced along the twisted placenta to the area of attachment in the uterus between the placenta and endometrium. The tips of the fingers were pressed between the endometrium and the chorion and the villi were detached, as the allantochorion gradually freed it was taken up by further twisting of the detached mass. The attachment was found firmer than usual may be the case resulted after abortion. The complete placenta was removed (Figure 2). Two boluses of antibiotics were placed in the uterus after removal of the placenta. The mare was parenterally administered with Inj. Intamox 4.5g (Intas Pharmaceutical), Inj. Gynotocin 50 IU [Svizera (ACE)], Inj. Anistamin 10ml (Intas Pharmaceutical) and Inj. Tetanus toxoid $5 \mathrm{ml}$ (Glaxo Smith Kline) intramuscularly. The owner was advised to repeat the antibiotic treatment for 3 days and to reduce the quantity of roughages and to eliminate the grains completely from the diet for a week. Mare recovered uneventfully after the treatment. Ishii [5] opined for the administration of Oxytocin at dosage of $50 \mathrm{IU}$ and uterine irrigation in mare placental retention. However, no attempt was made to lavage the uterus in the present case. Azawi and Taha [6] opined that neither flushing the uterus nor removal of the placenta is indicated in mares with retained placenta. They treated the retention of placenta in mare by antibiotics, Oxytocin injection and lavaged the uterus at 3rd day post foaling with normal saline and antibiotic. Arthur [7] suggested that manual removal of retained placenta in mare may results in hemorrhage, delayed uterine involution, endometrial damage and uterine prolapse. No such complications were observed in the present case. 


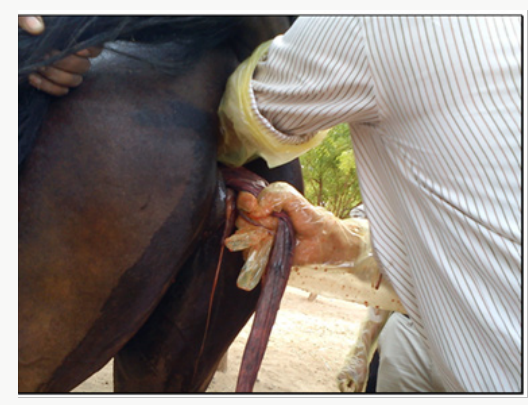

Figure 1: Twisting of placenta in rope fashion for manual removal.

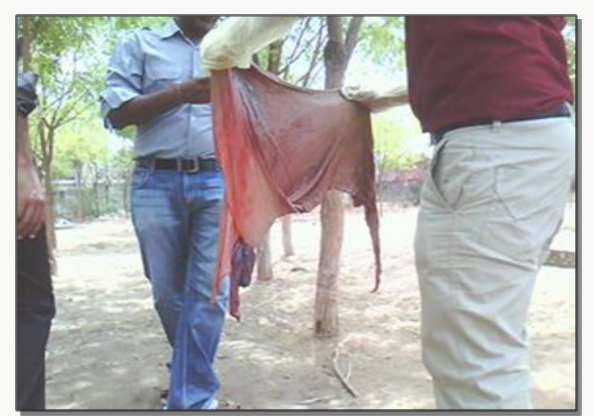

Figure 2: Manually removed placenta.

\section{References}

1. Vandeplassche M, Spincemaile J, Bouters R (1971) Aetiology, Pathogenesis and treatment of retained placenta in the mare. Equine Vet J 3(4): 144-147.

2. Blanchard TL, Bierschwal CJ, Youngquist RS (1983) Sequelae to percutaneous fetotomy in the mare. J Am Vet Med Assoc 182(10): 1127.

3. Provercher M, Threlfall WR, Murdrich P, W Keith Wearly (1988) Retained fetal membranes in the mare: A retrospective study. Can Vet J 29(11): 6776.

4. Allen WE (1988) Fertility and obstetrics in the Horse. $1^{\text {st }}$ ed. Boston: Blackwell Scientific, UK, pp.104.

5. Ishii M, Jitsukawa T, Shimamura T, Utsumi A, Endo M Yamanoi T (2007) Effect of placental retention time and associated treatments on reproductive performance in heavy draft horses. J equine vet Sci 19(2): 117-121.

6. Azawi OI, Taha MB (2008) Retention of fetal membranes in an Arabian mare: a case report. Iraqi Journal of Veterinary Sciences 22(1): 57-58.

7. Arthur GH, Noakes DE, Pearson H (1989) Veterinary Reproduction and Obstetrics, $6^{\text {th }}$ edn. Bailliere Tindall, London, UK, pp. 292.

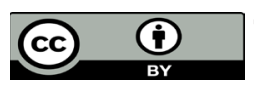

This work is licensed under Creative Commons Attribution 4.0 License

Submission Link:

Submit Article

DOI: 10.32474/CDVS.2018.01.000119

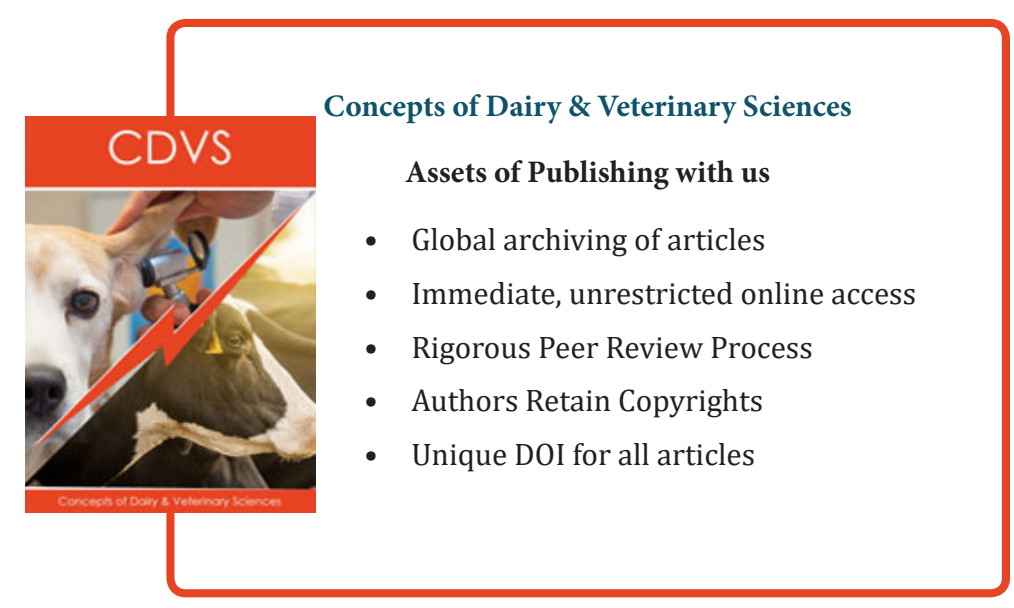

